Karl-Franzens Universität Graz

Technische Universität Graz

Medizinische Universität Graz

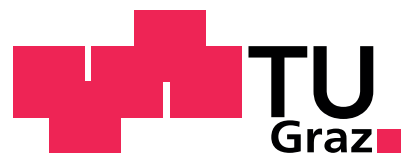

\title{
ON COMPUTATION OF THE SHAPE HESSIAN OF THE COST FUNCTIONAL WITHOUT SHAPE SENSITIVITY OF THE STATE VARIABLE
}
H. Kasumba
K. Kunisch 
SFB sponsors:

- Austrian Science Fund (FWF)

- University of Graz

- Graz University of Technology

- Medical University of Graz

- Government of Styria

- City of Graz

Das Land

Steiermark

Stadt $\mathbf{G} \mathbf{R}$ A Z $\mathbf{Z}$ Wissenschaft 


\title{
ON COMPUTATION OF THE SHAPE HESSIAN OF THE COST FUNCTIONAL WITHOUT SHAPE SENSITIVITY OF THE STATE VARIABLE
}

\author{
H. KASUMBA AND K. KUNISCH
}

\begin{abstract}
A framework for calculating the shape Hessian for the domain optimization problem with a partial differential equation as the constraint is presented. First and second order approximations of the cost with respect to geometry perturbations are arranged in an efficient manner that allows the computation of the shape derivative and Hessian of the cost without the necessity to involve the shape derivative of the state variable. In doing so, the state and adjoint variables are only required to be Hölder continuous with respect to geometry perturbations.
\end{abstract}

\section{INTRODUCTION}

Many important questions arise in the study of shape optimization problems. For instance, the existence and stability of optimal domains [HP91], the analysis of convergence of fixed point methods for free boundary problems [Til97], and speeding up of gradient type methods in shape optimization problems where the topological structure of the shape changes during iteration [Bur04]. All these questions are obviously linked to the second order information of the shape functional. Since the pioneering work of Fujii [Fuj87], the computation of second order shape derivatives has received a growing amount of attention, see for instance [DZ91a, Sim89, DZ91b, HP91, Til97]. In some of these contributions, the characterization of the shape Hessian is given only in a formal manner. The approach taken by Fujii [Fuj87] and Simon [Sim89] involves differentiation of the state equation with respect to the domain. The state variable varies in a Hilbert space $X$ which depends on the geometry with respect to which optimization is carried out. To obtain sensitivity information of $\Omega \mapsto \hat{J}(\Omega)=J(\Omega, u(\Omega))$, a chain rule approach involving the shape derivative of $\Omega \mapsto u(\Omega)$ is chosen. The rigorous analysis of this intermediate step is a non-trial task and as shown in Ito et al [IKP08], there are cases where the assumptions of this paper are applicable, while shape differentiability of the state does not hold. Other techniques that bypass the computation of the derivative of the state with respect to the domain are presented by Delfour and Zolesio in [DZ91a] and [DZ91b]. In [DZ91a, DZ91b], they use function space parametrization and function space embedding methods, respectively, to characterize the shape Hessian

Key words and phrases. Shape derivative, shape Hessian, Cost functional.

The second author was supported in part by the Austrian Science Fund Fond zur Forderung der Wissenschaftlichen Forschung (FWF) under grant SFB F32 (SFB "Mathematical Optimization and Applications in Biomedical Sciences"). 
of the cost functional. However, these techniques depend strongly on sophisticated differentiability properties of saddle point problems. In this paper, we present a computation of the shape derivative of $J$ under minimal regularity assumptions. The technique we employ was first suggested in [IKP06] for computing the first order information, and allows to compute the shape derivative of the mapping $\Omega \mapsto \hat{J}(\Omega)$ without using the shape derivative of the state variable with respect to the geometry. The method and the associated computation we present is general and is applicable to a large class of boundary value problems. However, to make the exposition more transparent, we present the results on a simple example rather than give a general exhaustive theory. As an example, we consider the following shape optimization problem:

$$
\min _{\Omega \in \mathcal{U}_{a d}} J(\Omega)=\frac{1}{2} \int_{\Omega}\left|y-y_{d}\right|^{2} d \Omega
$$

subject to

$$
\left\{\begin{array}{l}
-\Delta y=f \text { in } \Omega \\
y=0 \text { on } \Gamma
\end{array}\right.
$$

Here $\Omega$ is a domain with boundary $\Gamma:=\partial \Omega$ of class $C^{2,1}$. We shall denote by $H^{m}(\mathcal{S}), m \in \mathbb{R}$, the standard Sobolev space of order $m$ defined by

$$
H^{m}(\mathcal{S}):=\left\{u \in L^{2}(\mathcal{S}) \mid D^{\alpha} u \in L^{2}(\mathcal{S}) \text {, for } 0 \leq|\alpha| \leq m\right\},
$$

where $D^{\alpha}$ is the weak (or distributional) partial derivative, and $\alpha$ is a multi-index. Here $\mathcal{S}$, which is either the flow domain $\Omega$, or its boundary $\Gamma$, or part of its boundary. The norm $\|\cdot\|_{H^{m}(\mathcal{S})}$ associated with $H^{m}(\mathcal{S})$ is given by

$$
\|u\|_{H^{m}(\mathcal{S})}^{2}=\sum_{|\alpha| \leq m} \int_{\mathcal{S}}\left|D^{\alpha} u\right|^{2} d \mathcal{S} .
$$

Note that $H^{0}(\mathcal{S})=L^{2}(\mathcal{S})$ and $\|\cdot\|_{H^{0}(\mathcal{S})}=\|\cdot\|_{L^{2}(\mathcal{S})}$.

The data $f$ is assumed to belong to the space $H^{s}(\mathcal{D})$, where $\mathcal{D}$ is a bounded hold all domain, and $s \geq 0$ will be specified later on. We shall say that $y$ is the state and (1.2) is the state equation. The desired state $y_{d}$ in $(1.1)$ is assumed to be in $H^{1}(\mathcal{D})$. Our objective is to compute the first and second order derivatives of the cost $J$ with respect to $\Omega$ without the necessity of involving the derivative of the state $y$ with respect to $\Omega$.

\section{Definitions}

2.1. Shape derivative. Shapes are difficult entities to be dealt with directly, so we manipulate them by means of transformations. If $\Omega$ is the initial admissible shape, and $\Omega_{t}$ is the shape at time $t$, one considers transformations $T_{t}: \Omega \mapsto \Omega_{t}$. Such transformations can be constructed, for instance, by perturbation of the identity 
[DZ01]. To construct an admissible class of these transformations, let $\Omega \subset \overline{\mathcal{D}}$ be a bounded domain, and let

$$
\mathcal{T}_{a d}=\left\{\mathbf{V} \in C^{2,1}(\overline{\mathcal{D}}):\left.\mathbf{V}\right|_{\partial \mathcal{D}}=0\right\}
$$

be the space of deformation fields. The fields $\mathbf{V} \in \mathcal{T}_{a d}$ define for $t>0$, a perturbation of $\Omega$ by means of

$$
\begin{aligned}
T_{t}: \Omega & \mapsto \Omega_{t}(\mathbf{V}), \\
x & \mapsto T_{t}(x)=x+t \mathbf{V}(x) .
\end{aligned}
$$

For each $\mathbf{V} \in \mathcal{T}_{a d}$, there exists $\tau>0$ such that $T_{t}(\mathcal{D})=\mathcal{D}$ and $\left\{T_{t}\right\}$ is a family of $C^{2,1}$-diffeomorphisms for $|t|<\tau$ [DZ01]. For each $t \in \mathbb{R}$ with $|t|<\tau$, we set $\Omega_{t}=T_{t}(\Omega), \quad \Gamma_{t}=T_{t}(\Gamma)$. Thus $\Omega_{0}=\Omega, \Gamma_{0}=\Gamma, \Omega_{t} \subset \mathcal{D}$.

Let $\mathcal{J}=[0, \tau]$ with $\tau$ sufficiently small. Then the following regularity properties of the transformation $T_{t}$ can be shown, see for example ([IKP08, SZ92], [DZ01, Chapter 7] ):

$$
\begin{array}{ll}
T_{0}=i d & t \mapsto T_{t} \in C^{1}\left(\mathcal{J}, C^{1}\left(\overline{\mathcal{D}} ; \mathbb{R}^{d}\right)\right) \\
t \mapsto T_{t}^{-1} \in C\left(\mathcal{J}, C^{1}\left(\overline{\mathcal{D}} ; \mathbb{R}^{d}\right)\right) & t \mapsto I_{t} \in C^{1}(\mathcal{J}, C(\overline{\mathcal{D}})) \\
t \mapsto\left(D T_{t}\right)^{-T} \in C^{1}\left(\mathcal{J}, C\left(\overline{\mathcal{D}} ; \mathbb{R}^{d \times d}\right)\right) & \left.\frac{d}{d t} T_{t}\right|_{t=0}=\mathbf{V} \\
\left.\frac{d}{d t} T_{t}^{-1}\right|_{t=0}=-\mathbf{V} & \left.\frac{d}{d t} D T_{t}\right|_{t=0}=D \mathbf{V} \\
\left.\frac{d}{d t} D T_{t}^{-1}\right|_{t=0}=-D \mathbf{V} & \left.\frac{d}{d t} I_{t}\right|_{t=0}=\operatorname{div} \mathbf{V} \\
\left.I_{t}\right|_{t=0}=1 & \left.I_{t}^{-1}\right|_{t=0}=1 \\
\left.\frac{d}{d t} A(t)\right|_{t=0}=\operatorname{div} \mathbf{V}-\left(D \mathbf{V}+(D \mathbf{V})^{T}\right), &
\end{array}
$$

where

$$
I_{t}:=\operatorname{det} D T_{t}, \quad A(t):=I_{t}\left(D T_{t}\right)^{-1}\left(D T_{t}\right)^{-T},
$$

and the limits defining the derivatives at $t=0$ exist uniformly in $x \in \overline{\mathcal{D}}$.

Definition 2.1. For given $\mathbf{V} \in \mathcal{T}_{\text {ad }}$, the Eulerian derivative of $J$ at $\Omega$ in the direction $\mathbf{V}$ is defined as

$$
d J(\Omega) \mathbf{V}=\lim _{t \rightarrow 0^{+}} \frac{J\left(\Omega_{t}(\mathbf{V})\right)-J(\Omega)}{t} .
$$

The functional $J$ is said to be shape differentiable at $\Omega$ if $d J(\Omega) \mathbf{V}$ exists for all $\mathbf{V} \in \mathcal{T}_{a d}$ and the mapping $\mathbf{V} \mapsto d J(\Omega) \mathbf{V}$ is linear and continuous on $\mathcal{T}_{a d}$. If $J$ is shape differentiable, then there exists a distribution $\mathcal{G}$ in $\mathcal{T}_{a d}^{*}$ such that

$$
d J(\Omega) \mathbf{V}=\langle\mathcal{G}, \mathbf{V}\rangle_{\mathcal{T}_{a d}^{*} \times \mathcal{T}_{a d}} .
$$

The distribution $\mathcal{G}$ that is uniquely defined by (2.4) is called the shape gradient of $J$ at $\Omega$. 
2.2. Shape Hessian. Let $\mathbf{V}$ and $\mathbf{W}$ be given vector fields in $\mathcal{T}_{a d}$. As in the previous subsection, we associate with $\mathbf{V}$ and $\mathbf{W}$ the transformed domains $\Omega_{t}(\mathbf{V})$ and $\Omega_{t}(\mathbf{W})$.

Definition 2.2. [DZ01] Assume that $d J\left(\Omega_{t}(\mathbf{W})\right) \mathbf{V}$ exists for all $t \in[0, \tau]$. Then the functional $J$ is said to have a second order Eulerian semi-derivative at $\Omega$ in directions $(\mathbf{V}, \mathbf{W})$ if the following limit exists

$$
d^{2} J(\Omega)(\mathbf{V}, \mathbf{W})=\lim _{t \rightarrow 0^{+}} \frac{d J\left(\Omega_{t}(\mathbf{W})\right) \mathbf{V}-d J(\Omega) \mathbf{V}}{t} .
$$

The functional $J$ is said to be twice shape differentiable at $\Omega$ if for all $\mathbf{V}, \mathbf{W} \in \mathcal{T}_{\text {ad }}$ the second Eulerian semi-derivative $d^{2} J(\Omega)(\mathbf{V}, \mathbf{W})$ exists and the mapping

$$
(\mathbf{V}, \mathbf{W}) \mapsto d^{2} J(\Omega)(\mathbf{V}, \mathbf{W}): \mathcal{T}_{a d} \times \mathcal{T}_{a d} \mapsto \mathbb{R}
$$

is bilinear and continuous.

The distribution associated with the mapping in (2.6) is called the shape Hessian. It will be shown that the shape Hessian has its support on the boundary of $\Omega$ and that it is independent of the tangential component of $\mathbf{W}$ on the boundary. On the other hand, the tangential component of $\mathbf{V}$ contributes to the shape Hessian in general. Hence the shape Hessian is typically not symmetric.

\section{Shape DeRivative Via RE-ARRANGEMENT OF THE COST}

In this section we compute the shape derivative of $J$ in (1.1) by re-arranging the first perturbation of the cost with respect to the geometry. This result can be obtained using the general theory developed in Ito et al [IKP08]. However, we felt compelled to repeat some essential steps to give a basis for computing the second order information.

Using Definition 2.1, the first derivative can be expressed as

$$
d J(\Omega) \mathbf{V}=\lim _{t \rightarrow 0^{+}} \frac{1}{2 t}\left\{\int_{\Omega_{t}(\mathbf{V})}\left|y_{t}-y_{d}\right|^{2} d \Omega_{t}(\mathbf{V})-\int_{\Omega}\left|y-y_{d}\right|^{2} d \Omega\right\},
$$

where $y_{t}$ satisfies

$$
\left\{\begin{array}{l}
-\Delta y_{t}=f_{t} \text { in } \Omega_{t} \\
y_{t}=0 \text { on } \Gamma_{t}
\end{array}\right.
$$

We assume that

$$
f \in H^{1}(\mathcal{D}) \quad \text { and } \quad y_{d} \in H^{1}(\mathcal{D}) .
$$

The variational form of (3.2) is given by:

Find $y_{t} \in X_{t}:=H_{0}^{1}\left(\Omega_{t}\right)$ such that

$$
\left\langle\mathcal{E}\left(y_{t}^{t}, \Omega_{t}\right), \psi_{t}\right\rangle_{X_{t}^{*} \times X_{t}} \equiv\left(\nabla y_{t}, \nabla \psi_{t}\right)_{\Omega_{t}}-\left(f_{t}, \psi_{t}\right)_{\Omega_{t}}=0,
$$

holds for all $\psi_{t} \in X_{t}$.

Proposition 3.1. [GR86] There exists a unique solution $y_{t}$ to (3.2). Moreover, since the domain is assumed to be of class $C^{2,1}$, we have $y_{t} \in H^{3}\left(\Omega_{t}\right) \cap H_{0}^{1}\left(\Omega_{t}\right)$. 
Observe that at $t=0,\left.y_{t}\right|_{t=0}=y \in X,\left.\Omega_{t}\right|_{t=0}=\Omega,\left.X_{t}\right|_{t=0}=X$, and (3.3) becomes

$$
\langle\mathcal{E}(y, \Omega), \psi\rangle_{X^{*} \times X} \equiv(\nabla y, \nabla \psi)_{\Omega}-(f, \psi)_{\Omega}=0,
$$

which is the weak formulation of the state equation (1.2) with homogeneous Dirichlet boundary conditions. The functions $y_{t}$ and $y$ in (3.1) are defined on different domains. Therefore, to compute (3.1), one needs to transport $y_{t}$ back to $\Omega$. Any function $y_{t}: \Omega_{t} \mapsto \mathbb{R}^{2}$, can be mapped back to the reference domain by

$$
y^{t}=y_{t} \circ T_{t}: \Omega \mapsto \mathbb{R}^{2},
$$

where $\left(y_{t} \circ T_{t}\right)(x)=y_{t}\left(T_{t}(x)\right)$. Furthermore, the chain rule guarantees that the gradients of $y_{t}$ and $y^{t}$ are related by

$$
\left(\nabla y_{t}\right) \circ T_{t}=B_{t} \nabla y^{t},
$$

(see [SZ92] Prop: 2.29) where $B_{t}:=\left(D T_{t}\right)^{-T}$. Consequently, the transformation of (3.3) back to $\Omega$ is obtained as follows:

Find $y^{t} \in X$ such that

$$
\left\langle\tilde{\mathcal{E}}\left(y^{t}, t\right), \psi\right\rangle_{X^{*} \times X} \equiv\left(A(t) \nabla y^{t}, \nabla \psi\right)_{\Omega}-\left(f^{t} I_{t}, \psi\right)_{\Omega}=0 \quad \text { for all } \psi \in X .
$$

It is shown in $\left[\mathrm{HIK}^{+} 09\right]$ that for any $0<\alpha<1$, the following result

$$
(A(t) \xi, \xi) \geq \frac{\alpha}{2}|\xi|^{2}
$$

holds for $(\xi, x) \in \mathbb{R}^{2} \times \Omega$ and $\tau$ sufficiently small. Thus the bilinear form in (3.7) is elliptic uniformly in $t \in \mathcal{J}$. The adjoint state $p \in H_{0}^{1}(\Omega)$, is defined as the solution to

$$
\left\langle\mathcal{E}_{y}(y, \Omega) \psi, p\right\rangle_{X^{*} \times X}=\left(y-y_{d}, \psi\right)_{\Omega} \quad \text { for all } \psi \in H_{0}^{1}(\Omega)
$$

where

$$
\left\langle\mathcal{E}_{y}(y, \Omega) \psi, p\right\rangle_{X^{*} \times X}:=(\nabla \psi, \nabla p)_{\Omega} .
$$

Integrating the term $(\nabla \psi, \nabla p)_{\Omega}$, on the right hand side of (3.9) by parts, one obtains the strong form of the adjoint equation in (3.8), that we express as

$$
\left\{\begin{array}{l}
-\Delta p=y-y_{d} \text { in } \Omega, \\
p=0 \text { on } \Gamma .
\end{array}\right.
$$

The adjoint equation (3.10) possesses a unique solution $p \in H_{0}^{1}(\Omega)$. Moreover, since the domain is assumed to be of class $C^{2,1}$, we have that $p \in H^{3}(\Omega) \cap H_{0}^{1}(\Omega)$.

The existence of the primal and adjoint states allows the formulation of first order optimality conditions for problem (1.2). The following lemmas shall be utilized. 
Lemma 3.1. [IKP08]

(1) Let $g \in C\left(\mathcal{J}, W^{1,1}(\mathcal{D})\right)$, and assume that $\frac{\partial g}{\partial t}(0)$ exists in $L^{1}(\mathcal{D})$. Then

$$
\left.\frac{d}{d t} \int_{\Omega_{t}} g(t, x) d \Omega_{t}\right|_{t=0}=\int_{\Omega} \frac{\partial g}{\partial t}(0, x) d \Omega+\int_{\Gamma} g(0, x) \mathbf{V} \cdot \mathbf{n} d \Gamma .
$$

(2) Let $g \in C\left(\mathcal{J}, W^{2,1}(\mathcal{D})\right)$, and assume that $\frac{\partial g}{\partial t}(0)$ exists in $W^{1,1}(\mathcal{D})$. Then

$$
\left.\frac{d}{d t} \int_{\Gamma_{t}} g(t, x) d \Omega_{t}\right|_{t=0}=\int_{\Gamma} \frac{\partial g}{\partial t}(0, x) d \Gamma+\int_{\Gamma}\left(\frac{\partial g(0, x)}{\partial \mathbf{n}}+\kappa g(0, x)\right) \mathbf{V} \cdot \mathbf{n} d \Gamma,
$$

where $\kappa$ stands for the mean curvature of $\Gamma$.

The assumptions of Lemma 3.1 can be verified using the following result.

Lemma 3.2. [SZ92, Chapter.2]

(1) If $y \in L^{p}(\mathcal{D})$, then $t \mapsto y \circ T_{t}^{-1} \in C\left(\mathcal{J}, L^{p}(\mathcal{D})\right), 1 \leq p<\infty$.

(2) If $y \in H^{2}(\mathcal{D})$, then $t \mapsto y \circ T_{t}^{-1} \in C\left(\mathcal{J}, H^{2}(\mathcal{D})\right.$ ).

(3) If $y \in H^{2}(\mathcal{D})$, then $\left.\frac{d}{d t}\left(y \circ T_{t}^{-1}\right)\right|_{t=0}$ exists in $H^{1}(\mathcal{D})$ and is given by

$$
\left.\frac{d}{d t}\left(y \circ T_{t}^{-1}\right)\right|_{t=0}=-(D y) \mathbf{V} \text {. }
$$

Remark 3.1. As a consequence of Lemma 3.2, we note that $\left.\frac{d}{d t} \nabla\left(y \circ T_{t}^{-1}\right)\right|_{t=0}$ exists in $L^{2}(\mathcal{D})$ and is given by

$$
\left.\frac{d}{d t} \nabla\left(y \circ T_{t}^{-1}\right)\right|_{t=0}=-\nabla(D y \mathbf{V})
$$

For the transformation of domain integrals, the following well known fact will be used repeatedly.

Lemma 3.3. Let $\phi_{t} \in L^{1}\left(\Omega_{t}\right)$, then $\phi_{t} \circ T_{t} \in L^{1}(\Omega)$ and

$$
\int_{\Omega_{t}} \phi_{t} d \Omega_{t}=\int_{\Omega} \phi_{t} \circ T_{t} I_{t} d \Omega
$$

Lemma 3.4. [IKP06] For any $f \in L^{p}(\mathcal{D}), p \geq 1$, we have $\lim _{t \rightarrow 0} f \circ T_{t}=f$ in $L^{p}(\mathcal{D})$.

\subsection{Preliminary results.}

Lemma 3.5. There exists a unique solution $y^{t} \in H_{0}^{1}(\Omega) \cap H^{2}(\Omega)$ to (3.7) for $t>0$ sufficiently small. Moreover,

$$
\lim _{t \rightarrow 0^{+}} \frac{\left\|y^{t}-y\right\|_{H^{2}(\Omega)}}{|t|^{\frac{1}{2}}}=0,
$$

holds, where $y$ is the weak solution of (1.2). 
Proof. The existence and uniqueness of a solution to (3.7) is established in [DZ01, Pg. 396]. Therefore, it suffices to show the second statement. Subtracting equation (3.7) from (3.4), one obtains

$$
\begin{aligned}
\left(A(t) \nabla\left(y^{t}-y\right), \nabla \psi\right)_{\Omega} & =-((A(t)-I) \nabla y, \nabla \psi)_{\Omega}+\left(f^{t} I_{t}-f, \psi\right)_{\Omega} \\
& =(\operatorname{div}((A(t)-I) \nabla y), \psi)_{\Omega}+\left(f^{t} I_{t}-f, \psi\right)_{\Omega} .
\end{aligned}
$$

We have that $y^{t}-y$ belongs to $H_{0}^{1}(\Omega)$. By standard elliptic regularity theory [Eva98, Pg.317] we obtain that $y^{t}-y \in H_{0}^{1}(\Omega) \cap H^{2}(\Omega)$ and

$$
\left\|\frac{1}{t}\left(y^{t}-y\right)\right\|_{H^{2}(\Omega)} \leq C \wp(t)
$$

where $\wp(t):=\left\{\left\|\operatorname{div}\left(\frac{1}{t}(A(t)-I) \nabla y\right)\right\|_{L^{2}(\Omega)}+\left\|\frac{1}{t}\left(f^{t} I_{t}-f\right)\right\|_{L^{2}(\Omega)}\right\}$ and $C$ is some appropriate constant which may be chosen independently of $t$. Following [HIK $\left.{ }^{+} 09\right]$, we can show that the right hand side in (3.1) is bounded uniformly in $t$. Consequently,

$$
\lim _{t \rightarrow 0^{+}} \frac{1}{\sqrt{t}}\left\|y^{t}-y\right\|_{H^{2}(\Omega)} \leq C \lim _{t \rightarrow 0^{+}} \wp(t) \sqrt{t}=0
$$

which implies (3.11).

By linearity of $\mathcal{E}$ in $y$, we have

$$
\left\langle\mathcal{E}(v, \Omega)-\mathcal{E}(y, \Omega)-\mathcal{E}_{y}(y, \Omega)(v-y), \psi\right\rangle_{X^{*} \times X}=0, \text { for all } v, \psi \in X .
$$

Lemma 3.6. The following result holds

$$
\lim _{t \rightarrow 0^{+}} \frac{1}{t}\left\langle\tilde{\mathcal{E}}\left(y^{t}, t\right)-\tilde{\mathcal{E}}(y, t)-\left(\mathcal{E}\left(y^{t}, \Omega\right)-\mathcal{E}(y, \Omega)\right), \psi\right\rangle_{X^{*} \times X}=0,
$$

for every $\psi \in X$.

Proof. Let

$$
\mathcal{G}(t):=\left\langle\tilde{\mathcal{E}}\left(y^{t}, t\right)-\tilde{\mathcal{E}}(y, t)-\left(\mathcal{E}\left(y^{t}, \Omega\right)-\mathcal{E}(y, \Omega)\right), \psi\right\rangle_{X^{*} \times X} .
$$

Then $\mathcal{G}(t)=\left[\left(I_{t} B_{t} \nabla\left(y^{t}-y\right), B_{t} \psi\right)_{\Omega}-\left(\nabla\left(y^{t}-y\right), \psi\right)_{\Omega}\right]$, and $\lim _{t \rightarrow 0^{+}} \frac{1}{t} \mathcal{G}(t)=0$ follows from Lemma 3.5 and the differentiability of the mappings $t \mapsto I_{t}, t \mapsto B_{t}$.

Theorem 3.1. Let $y$ and $p$ be the solutions to (1.2) and (3.10), respectively. Then

$$
\left.\frac{d}{d t}\langle\tilde{\mathcal{E}}(y, t), p\rangle_{X^{*} \times X}\right|_{t=0}=-\int_{\Gamma}\left[\frac{\partial y}{\partial \mathbf{n}} \frac{\partial p}{\partial \mathbf{n}}\right] \mathbf{V} \cdot \mathbf{n} d \Gamma-\int_{\Omega}\left(y-y_{d}\right) \nabla y \cdot \mathbf{V} d \Omega .
$$

Proof. The proof is a minor modification of a similar result in [IKP08]. Since $\Omega \in$ $C^{2,1}, y$ and $p$ can be extended to functions in $H^{2}(\mathcal{D})$ which we again denote by the same symbol. Furthermore, observe that

$$
\langle\tilde{\mathcal{E}}(y, t), p\rangle_{X^{*} \times X} \equiv(A(t) \nabla y, \nabla p)_{\Omega}-\left(f^{t} I_{t}, p\right)_{\Omega}
$$

can be mapped back to $\Omega_{t}$ to obtain

$$
\langle\tilde{\mathcal{E}}(y, t), p\rangle_{X^{*} \times X}=\left(\nabla\left(y \circ T_{t}^{-1}\right), \nabla\left(p \circ T_{t}^{-1}\right)\right)_{\Omega_{t}}-\left(f,\left(p \circ T_{t}^{-1}\right)\right)_{\Omega_{t}} .
$$


Thus, Lemma 3.1(1) and Lemma 3.2 imply that

$$
\left.\frac{d}{d t}\langle\tilde{\mathcal{E}}(y, t), p\rangle_{X^{*} \times X}\right|_{t=0}=\int_{\Omega}(\nabla \dot{y} \cdot \nabla p+\nabla y \cdot \nabla \dot{p}-f \dot{p}) d \Omega+\int_{\Gamma}(\nabla y \cdot \nabla p) \mathbf{V} \cdot \mathbf{n} d \Gamma,
$$

where $\dot{y}=-\nabla y \cdot \mathbf{V}, \dot{p}=-\nabla p \cdot \mathbf{V}$. Note $\dot{y}$ as well as $\dot{p}$ do not belong to $H_{0}^{1}(\Omega)$ but they are elements of $H^{1}(\Omega)$. Applying Greens theorem implies

$$
\begin{aligned}
\left.\frac{d}{d t}\langle\tilde{\mathcal{E}}(y, t), p\rangle_{X^{*} \times X}\right|_{t=0}= & \int_{\Omega}(-\Delta y-f) \dot{p} d \Omega+\int_{\Gamma}\left(\frac{\partial y}{\partial \mathbf{n}} \dot{p}+\dot{y} \frac{\partial p}{\partial \mathbf{n}}+\nabla y \cdot \nabla p \mathbf{V} \cdot \mathbf{n}\right) d \Gamma \\
& +\int_{\Omega}(-\Delta p) \dot{y} d \Omega .
\end{aligned}
$$

Since $y, p \in \mathbf{H}_{0}^{1}(\Omega)$, we have

$$
\int_{\Gamma}\left(\frac{\partial y}{\partial \mathbf{n}} \dot{p}+\dot{y} \frac{\partial p}{\partial \mathbf{n}}+\nabla y \cdot \nabla p \mathbf{V} \cdot \mathbf{n}\right) d \Gamma=-\int_{\Gamma}\left[\frac{\partial y}{\partial \mathbf{n}} \frac{\partial p}{\partial \mathbf{n}}\right] \mathbf{V} \cdot \mathbf{n} d \Gamma .
$$

The strong forms of the state (1.2) and adjoint (3.10) imply

$$
\left.\frac{d}{d t}\langle\tilde{\mathcal{E}}(y, t), p\rangle_{X^{*} \times X}\right|_{t=0}=-\int_{\Gamma}\left[\frac{\partial y}{\partial \mathbf{n}} \frac{\partial p}{\partial \mathbf{n}}\right] \mathbf{V} \cdot \mathbf{n} d \Gamma-\left(y-y_{d}, \nabla y \cdot \mathbf{V}\right)_{\Omega}
$$

3.2. Shape derivative. In this subsection we establish the expression for the shape derivative for the cost functional $J$. Since $y-y_{d} \in L^{2}(\Omega)$, the cost functional $J(\Omega)$ is well defined. The associated adjoint state $p \in X$ is given as a solution to (3.10).

Theorem 3.2. The shape derivative of $J(y, \Omega)$ exists and it is given by the expression

$$
d J(\Omega) \mathbf{V}=\int_{\Gamma}\left[\frac{\partial y}{\partial \mathbf{n}} \frac{\partial p}{\partial \mathbf{n}}+\frac{1}{2}\left(y-y_{d}\right)^{2}\right] \mathbf{V} \cdot \mathbf{n} d \Gamma .
$$

Proof. The general result in [IKP08] can be utilized to derive the expression in (3.14). However, we provide the proof here in a more elegant way than earlier in [IKP08]. Let $\boldsymbol{\Delta}_{1}:=J\left(y_{t}, \Omega_{t}\right)-J(y, \Omega)$. Then

$$
\boldsymbol{\Delta}_{1}=\int_{\Omega}\left(I_{t} \frac{1}{2}\left(y^{t}-y_{d}\right)^{2}-\frac{1}{2}\left(y-y_{d}\right)^{2}\right) d \Omega .
$$

We can express $\boldsymbol{\Delta}_{1}$ as $\boldsymbol{\Delta}_{1}=\boldsymbol{\Delta}_{1,1}(t)+\boldsymbol{\Delta}_{1,2}(t)+\boldsymbol{\Delta}_{1,3}(t)+\boldsymbol{\Delta}_{1,4}(t)$, where

$$
\begin{aligned}
& \boldsymbol{\Delta}_{1,1}(t)=\int_{\Omega} I_{t}\left[\frac{1}{2}\left(y^{t}-y_{d}\right)^{2}-\frac{1}{2}\left(y-y_{d}\right)^{2}-\left(y-y_{d}, y^{t}-y\right)\right] d \Omega, \\
& \boldsymbol{\Delta}_{1,2}(t)=\int_{\Omega}\left(I_{t}-1\right)\left(y-y_{d}, y^{t}-y\right) d \Omega, \quad \boldsymbol{\Delta}_{1,3}(t)=\int_{\Omega}\left(y-y_{d}, y^{t}-y\right) d \Omega, \\
& \boldsymbol{\Delta}_{1,4}(t)=\int_{\Omega}\left(I_{t}-1\right) \frac{1}{2}\left(y-y_{d}\right)^{2} d \Omega .
\end{aligned}
$$


From (3.15) and the embedding of $H_{0}^{1}(\Omega)$ into $L^{2}(\Omega)$, it follows that

$$
\left|\boldsymbol{\Delta}_{1,1}(t)\right| \leq K|| y^{t}-y \|_{H^{1}(\Omega)}^{2},
$$

where $K>0$ does not depend on $t \in \mathcal{J}$. Using Lemma 3.5 and Lemma 4.1, we have that

$$
\lim _{t \rightarrow 0} \frac{1}{t} \Delta_{1,1}(t)=0
$$

Next, observe that

$$
\left|\frac{\boldsymbol{\Delta}_{1,2}(t)}{t}\right| \leq K\left\|\frac{\left(I_{t}-I\right)}{t}\right\|_{L^{\infty}}\left\|y-y_{d}\right\|_{H^{1}(\Omega)}\left\|y^{t}-y\right\|_{H^{1}(\Omega)}
$$

Therefore, by Lemma 3.5 and (2.1), one obtains $\lim _{t \rightarrow 0}\left|\frac{1}{t} \Delta_{1,2}(t)\right|=0$. Using the adjoint equation (3.8) with $\psi=y^{t}-y \in X$ we have that

$$
\begin{aligned}
\Delta_{1,3}(t) & =\left\langle\mathcal{E}_{y}(y, \Omega)\left(y^{t}-y\right), p\right\rangle_{X^{*} \times X} \\
& =-\langle\tilde{\mathcal{E}}(y, t)-\tilde{\mathcal{E}}(y, 0), p\rangle_{X^{*} \times X} \\
& -\left\langle\mathcal{E}\left(y^{t}, \Omega\right)-\mathcal{E}(y, \Omega)-\mathcal{E}_{y}(y, \Omega)\left(y^{t}-y\right), p\right\rangle_{X^{*} \times X} \\
& -\left\langle\tilde{\mathcal{E}}\left(y^{t}, t\right)-\tilde{\mathcal{E}}(y, t)-\mathcal{E}\left(y^{t}, \Omega\right)+\mathcal{E}(y, \Omega), p\right\rangle_{X^{*} \times X} .
\end{aligned}
$$

By Lemma 3.5, equation (3.12), and Lemma 3.6, we find

$$
\begin{aligned}
\lim _{t \rightarrow 0} \frac{\Delta_{1,3}(t)}{t} & =-\left.\frac{d}{d t}\langle\tilde{\mathcal{E}}(y, t), p\rangle_{X^{*} \times X}\right|_{t=0}, \\
& =\int_{\Gamma} \frac{\partial y}{\partial \mathbf{n}} \frac{\partial p}{\partial \mathbf{n}} \mathbf{V} \cdot \mathbf{n} d \Gamma+\int_{\Omega}\left(y-y_{d}\right) \nabla y \cdot \mathbf{V} d \Omega,
\end{aligned}
$$

where we use (3.13). Since $y \in H^{2}(\Omega)$, it follows that $\frac{1}{2}\left(y-y_{d}\right)^{2} \in W^{1,1}(\Omega)$. This implies that $\frac{d}{d t}\left[\frac{1}{2}\left(y \circ T_{t}^{-1}-y_{d}\right)^{2}\right]_{t=0}$ exists in $L^{1}(\Omega)$, [SZ92, Pg.65]. Hence, since $\Delta_{1,4}(t)=\int_{\Omega_{t}} \frac{1}{2}\left(y \circ T_{t}^{-1}-y_{d}\right)^{2} d \Omega_{t}-\int_{\Omega} \frac{1}{2}\left(y-y_{d}\right)^{2} d \Omega$, we have by Lemma 3.1(1), that

$$
\begin{aligned}
\lim _{t \rightarrow 0} \frac{\Delta_{1,4}(t)}{t} & =\left.\frac{d}{d t} \int_{\Omega_{t}} \frac{1}{2}\left(y \circ T_{t}^{-1}-y_{d}\right)^{2}\right|_{t=0} d \Omega_{t}, \\
& =\int_{\Omega} \frac{d}{d t}\left[\frac{1}{2}\left(y \circ T_{t}^{-1}-y_{d}\right)^{2}\right]_{t=0} d \Omega+\int_{\Gamma} \frac{1}{2}\left(y-y_{d}\right)^{2} \mathbf{V} \cdot \mathbf{n} d \Gamma \\
& =-\int_{\Omega}\left(y-y_{d}\right) \nabla y \cdot \mathbf{V} d \Omega+\int_{\Gamma} \frac{1}{2}\left(y-y_{d}\right)^{2} \mathbf{V} \cdot \mathbf{n} d \Gamma
\end{aligned}
$$

Hence, (3.16) and (3.17) yield the desired expression for the shape derivative. 


\section{Shape Hessian via Re-ARRAngement of the COST}

In this section we compute the shape Hessian of $J$ in (1.1) by re-arranging the second order perturbation of the cost with respect to the geometry. Using the divergence theorem, we can express the first derivative derived in the previous section as follows

$$
d J(\Omega) \mathbf{V}=\int_{\Omega} \operatorname{div}\left(\left[\frac{1}{2}\left(y_{d}\right)^{2}+\nabla y \cdot \nabla p\right] \mathbf{V}\right) d \Omega .
$$

Using Definition 2.2, the second order Eulerian semi-derivative of $J$ at $\Omega$ in direction $(\mathbf{V}, \mathbf{W})$ can be expressed as

$$
d^{2} J(\Omega)(\mathbf{V}, \mathbf{W})=\lim _{t \rightarrow 0^{+}} \frac{d J\left(\Omega_{t}(\mathbf{W})\right) \mathbf{V}-d J(\Omega) \mathbf{V}}{t},
$$

where

$$
d J\left(\Omega_{t}(\mathbf{W})\right) \mathbf{V}=\int_{\Omega_{t}(\mathbf{W})} \operatorname{div}\left[\frac{1}{2} y_{d}^{2}+\nabla y_{t} \cdot \nabla p_{t}\right] \mathbf{V} d \Omega_{t}(\mathbf{W}),
$$

and $y_{t}, p_{t}$ satisfy

$$
\begin{array}{llllll}
-\Delta y_{t}=f_{t} \quad \text { in } \quad \Omega_{t}(\mathbf{W}), & y_{t}=0 & \text { on } & \Gamma_{t}(\mathbf{W}), \\
-\Delta p_{t}=y_{t}-y_{d} & \text { in } \quad \Omega_{t}(\mathbf{W}), & p_{t}=0 & \text { on } & \Gamma_{t}(\mathbf{W}) .
\end{array}
$$

The weak form of (4.3) is given by:

Find $p_{t} \in H_{0}^{1}\left(\Omega_{t}(\mathbf{W})\right)$ such that

$$
\left(\nabla p_{t}, \nabla \psi_{t}\right)_{\Omega_{t}(\mathbf{W})}=\left(y_{t}-y_{d}, \psi_{t}\right)_{\Omega_{t}(\mathbf{W})} \quad \text { for all } \quad \psi_{t} \in H_{0}^{1}\left(\Omega_{t}(\mathbf{W})\right) \text {. }
$$

The transformation of (4.4) back to $\Omega$ leads to the problem Find $p^{t} \in X$ such that

$$
\left(A(t) \nabla p^{t}, \nabla \psi\right)_{\Omega}-\left(\left(y^{t}-y_{d}\right) I_{t}, \psi\right)_{\Omega}=0 \quad \text { for all } \psi \in X .
$$

\subsection{Preliminary results.}

Lemma 4.1. There exists a unique solution $p^{t} \in H_{0}^{1}(\Omega) \cap H^{2}(\Omega)$ to (4.5) for $t>0$ sufficiently small. Moreover,

$$
\lim _{t \rightarrow 0^{+}} \frac{\left\|p^{t}-p\right\|_{H^{2}(\Omega)}}{|t|^{\frac{1}{2}}}=0,
$$

holds, where $p$ is the weak solution of (3.10).

Proof. The existence and uniqueness of a solution to (4.5) can be established in an analogous was as in Lemma 3.5. Subtracting equation (4.5) from (4.4) at $t=0$, one obtains for $\psi \in H_{0}^{1}(\Omega)$

$$
\begin{aligned}
\left(A(t) \nabla\left(p^{t}-p\right), \nabla \psi\right)_{\Omega} & =-((A(t)-I) \nabla p, \nabla \psi)_{\Omega}+\left(\left(y^{t}-y_{d}\right) I_{t}-\left(y-y_{d}\right), \psi\right)_{\Omega} \\
& =(\operatorname{div}((A(t)-I) \nabla p), \psi)_{\Omega}+\left(\left(y^{t}-y_{d}\right) I_{t}-\left(y-y_{d}\right), \psi\right)_{\Omega} .
\end{aligned}
$$


We have that $p^{t}-p$ belongs to $H_{0}^{1}(\Omega)$. By standard elliptic regularity theory [Eva98, Pg.317] we obtain that $p^{t}-p \in H_{0}^{1}(\Omega) \cap H^{2}(\Omega)$ and

$$
\left\|\frac{1}{t}\left(p^{t}-p\right)\right\|_{H^{2}(\Omega)} \leq C_{\wp}(t)
$$

where $\wp(t):=\left\{\left\|\operatorname{div}\left(\frac{1}{t}(A(t)-I) \nabla p\right)\right\|_{L^{2}(\Omega)}+\| \frac{1}{t}\left(\left(y^{t}-y_{d}\right) I_{t}-\left(y-y_{d}\right) \|_{L^{2}(\Omega)}\right\}\right.$ and $C$ is some appropriate constant which may be chosen independently of $t$. Following $\left[\mathrm{HIK}^{+} 09\right]$, we can show that the term $\left\|\operatorname{div}\left(\frac{1}{t}(A(t)-I) \nabla p\right)\right\|_{L^{2}(\Omega)}$ is bounded uniformly in $t$. Furthermore, Lemma 3.5 also suggests that $\| \frac{1}{t}\left(\left(y^{t}-y_{d}\right) I_{t}-\left(y-y_{d}\right) \|_{L^{2}(\Omega)}\right.$ is bounded uniformly in $t$. Consequently,

$$
\lim _{t \rightarrow 0^{+}} \frac{1}{\sqrt{t}}\left\|p^{t}-p\right\|_{H^{2}(\Omega)} \leq C \lim _{t \rightarrow 0^{+}} \wp(t) \sqrt{t}=0
$$

which implies (4.6).

For the computation of the second order derivative, presence of $y$ and $p$ as states suggests the introduction of two adjoint states $(\hat{\Sigma}, \hat{P}) \in H^{2}(\Omega) \times H^{2}(\Omega)$. Following [DZ91b], we introduce $(\hat{\Sigma}, \hat{P})$ as solutions to the equations

$$
\begin{array}{r}
\int_{\Omega} \operatorname{div}([\nabla y \cdot \nabla \tilde{p}] \mathbf{V})+\Delta \tilde{p} \hat{\Sigma}+\tilde{p} \Delta \hat{\Sigma}+\nabla \tilde{p} \cdot \nabla \hat{\Sigma} d \Omega=0, \text { for all } \quad \tilde{p} \in H^{2}(\Omega), \\
\int_{\Omega} \operatorname{div}([\nabla \tilde{y} \cdot \nabla p] \mathbf{V})+\Delta \tilde{y} \hat{P}+\tilde{y} \Delta \hat{P}+\nabla \tilde{y} \cdot \nabla \hat{P}+\tilde{y} \hat{\Sigma} d \Omega=0, \text { for all } \tilde{y} \in H^{2}(\Omega) .
\end{array}
$$

By integrating the second order terms in the expressions above, we obtain

$$
\begin{array}{r}
\int_{\Omega} \operatorname{div}([\nabla y \cdot \nabla \tilde{p}] \mathbf{V})-\nabla \tilde{p} \cdot \nabla \hat{\Sigma} d \Omega+\int_{\Gamma} \frac{\partial \tilde{p}}{\partial \mathbf{n}} \hat{\Sigma}+\frac{\partial \hat{\Sigma}}{\partial \mathbf{n}} \tilde{p} d \Gamma=0 \text { for all } \tilde{p} \in \mathcal{V} \\
\int_{\Omega} \operatorname{div}([\nabla \tilde{y} \cdot \nabla p] \mathbf{V})-\nabla \tilde{y} \cdot \nabla \hat{P}+\tilde{y} \hat{\Sigma} d \Omega+\int_{\Gamma} \frac{\partial \tilde{y}}{\partial \mathbf{n}} \hat{P}+\frac{\partial \hat{P}}{\partial \mathbf{n}} \tilde{y} d \Gamma=0 \text { for all } \tilde{y} \in \mathcal{V}
\end{array}
$$

where $\mathcal{V}=H^{2}(\Omega)$. Note that multiplying (1.2) and (3.10) by $\hat{P} \in \mathcal{V}$ and $\hat{\Sigma} \in \mathcal{V}$, respectively, one obtains

$$
\begin{array}{rll}
\langle E(y, \Omega), \hat{P}\rangle_{\Upsilon}=0 & \text { for all } & \hat{P} \in \mathcal{V}, \\
\langle E(p, y, \Omega), \hat{\Sigma}\rangle_{\Upsilon}=0 & \text { for all } & \hat{\Sigma} \in \mathcal{V}
\end{array}
$$

where $\Upsilon:=\mathcal{V}^{*} \times \mathcal{V}$ and

$$
\begin{aligned}
\langle E(y, \Omega), \hat{P}\rangle_{\Upsilon} & \equiv \int_{\Omega}(f \hat{P}-\nabla y \cdot \nabla \hat{P}) d \Omega+\int_{\Gamma}\left(\frac{\partial y}{\partial \mathbf{n}} \hat{P}+y \frac{\partial \hat{P}}{\partial \mathbf{n}}\right) d \Gamma \\
\langle E(p, y, \Omega), \hat{\Sigma}\rangle_{\Upsilon} & \equiv \int_{\Omega}\left(\left(y-y_{d}\right) \hat{\Sigma}-\nabla p \cdot \nabla \hat{\Sigma}\right) d \Omega+\int_{\Gamma}\left(\frac{\partial p}{\partial \mathbf{n}} \hat{\Sigma}+\frac{\partial \hat{\Sigma}}{\partial \mathbf{n}} p\right) d \Gamma .
\end{aligned}
$$


Using the divergence theorem on the boundary terms in (4.8) and (4.9), one obtains the equations on $\Omega_{t}(\mathbf{W})$ as follows

$$
\begin{array}{rll}
\left\langle E\left(y_{t}, \Omega_{t}(\mathbf{W})\right), \hat{P}_{t}\right\rangle_{\Upsilon_{t}}=0 & \text { for all } & \hat{P}_{t} \in \mathcal{V}_{t}, \\
\left\langle E\left(p_{t}, y_{t}, \Omega_{t}(\mathbf{W})\right), \hat{\Sigma}_{t}\right\rangle_{\Upsilon_{t}}=0 & \text { for all } & \hat{\Sigma}_{t} \in \mathcal{V}_{t},
\end{array}
$$

where $\Upsilon_{t}:=\mathcal{V}_{t}^{*} \times \mathcal{V}_{t}$ and

$$
\begin{aligned}
\left\langle E\left(y_{t}, \Omega_{t}(\mathbf{W})\right), \hat{P}_{t}\right\rangle_{\Upsilon_{t}}:= & \int_{\Omega_{t}(\mathbf{W})} f_{t} \hat{P}_{t}-\nabla y_{t} \cdot \nabla \hat{P}_{t}+\operatorname{div}\left(\nabla y_{t} \hat{P}_{t}+\nabla \hat{P}_{t} y_{t}\right) d \Omega_{t}(\mathbf{W}), \\
\left\langle E\left(p_{t}, y_{t}, \Omega_{t}(\mathbf{W})\right), \hat{\Sigma}_{t}\right\rangle_{\Upsilon_{t}}:= & \int_{\Omega_{t}(\mathbf{W})}\left(y_{t}-y_{d}\right) \hat{\Sigma}_{t}-\nabla p_{t} \cdot \nabla \hat{\Sigma}_{t} d \Omega_{t}(\mathbf{W}) \\
& +\int_{\Omega_{t}(\mathbf{W})} \operatorname{div}\left(\nabla p_{t} \hat{\Sigma}_{t}+\nabla \hat{\Sigma}_{t} p_{t}\right) d \Omega_{t}(\mathbf{W}) .
\end{aligned}
$$

Transforming (4.10) back to $\Omega$, one obtains

$$
\begin{aligned}
\left\langle\tilde{E}\left(y^{t}, t\right), \hat{P}^{t}\right\rangle_{\Upsilon} & \equiv \int_{\Omega}\left(I_{t} f^{t} \hat{P}^{t}-A(t) \nabla y^{t} \cdot \nabla \hat{P}^{t}+I_{t}\left(B_{t}\right)_{k} \nabla \theta_{k}^{t}\right) d \Omega=0, \\
\left\langle\tilde{E}\left(p^{t}, y^{t}, t\right), \hat{\Sigma}^{t}\right\rangle_{\Upsilon} & \equiv \int_{\Omega}\left(I_{t}\left(y^{t}-y_{d}\right) \hat{\Sigma}^{t}-A(t) \nabla p^{t} \cdot \nabla \hat{\Sigma}^{t}+I_{t}\left(B_{t}\right)_{k} \nabla \vartheta_{k}^{t}\right) d \Omega=0,
\end{aligned}
$$

where

$$
\begin{aligned}
& \theta^{t}:=B_{t} \nabla y^{t} \hat{P}^{t}+B_{t} \nabla \hat{P}^{t} y^{t}, \quad \vartheta^{t}:=B_{t} \nabla p^{t} \hat{\Sigma}^{t}+B_{t} \nabla \hat{\Sigma}^{t} p^{t}, \quad \theta:=B_{t} \nabla y \hat{P}+B_{t} \nabla \hat{P} y, \\
& \theta_{0}:=\nabla y \hat{P}+\nabla \hat{P} y, \quad \theta^{r}=B_{t} \nabla y^{t} \hat{P}+B_{t} \nabla \hat{P} y^{t} .
\end{aligned}
$$

Lemma 4.2. Let

$$
\mathcal{W}(t):=\left\langle\tilde{E}\left(y^{t}, t\right)-\tilde{E}(y, t)-\left(E\left(y^{t}, \Omega\right)-E(y, \Omega)\right), \hat{P}\right\rangle_{\Upsilon}
$$

and

$$
\mathcal{S}(t):=\left\langle\tilde{E}\left(p^{t}, y^{t}, t\right)-\tilde{E}(p, y, t)-\left(E\left(p^{t}, y^{t}, \Omega\right)-E(p, y, \Omega)\right), \hat{\Sigma}\right\rangle_{\Upsilon} .
$$

Then

$$
\lim _{t \rightarrow 0^{+}} \frac{1}{t} \mathcal{W}(t)=0, \quad \lim _{t \rightarrow 0^{+}} \frac{1}{t} \mathcal{S}(t)=0
$$

Proof. It suffices to prove that $\lim _{t \rightarrow 0^{+}} \frac{1}{t} \mathcal{W}(t)=0$, since the second expression follows in an analogous way. Using (4.11) with $\hat{P}^{t}$ replaced by $\hat{P}$ and $\left(y^{t}, \hat{P}^{t}\right)$ replaced by $(y, \hat{P})$, we obtain

$$
\left\langle\tilde{E}\left(y^{t}, t\right), \hat{P}\right\rangle_{\Upsilon}=\int_{\Omega} I_{t} f^{t} \hat{P}-A(t) \nabla y^{t} \cdot \nabla \hat{P} d \Omega+\int_{\Omega} I_{t}\left(B_{t}\right)_{k} \nabla \theta_{k}^{r} d \Omega,
$$


and

$$
\langle\tilde{E}(y, t), \hat{P}\rangle_{\Upsilon}=\int_{\Omega} I_{t} f^{t} \hat{P}-A(t) \nabla y \cdot \nabla \hat{P} d \Omega+\int_{\Omega} I_{t}\left(B_{t}\right)_{k} \nabla \theta_{k} d \Omega,
$$

respectively. Subtracting (4.13) from (4.12), one obtains

$$
\left\langle\tilde{E}\left(y^{t}, t\right), \hat{P}\right\rangle_{\Upsilon}-\langle\tilde{E}(y, t), \hat{P}\rangle_{\Upsilon}=\int_{\Omega}-A(t) \nabla \delta y \cdot \nabla \hat{P}+I_{t}\left(B_{t}\right)_{k} \nabla\left(\theta_{k}^{r}-\theta_{k}\right) d \Omega,
$$

where $\delta y=y^{t}-y$ and $\delta p=p^{t}-p$. Replacing $y$ by $y^{t}$ in (4.8) and subtracting the result from (4.8), we obtain

$$
\left\langle E(y, \Omega)-E\left(y^{t}, \Omega\right), \hat{\Sigma}\right\rangle_{\Upsilon}=\int_{\Omega} \nabla \delta y \cdot \nabla \hat{P} d \Omega-\int_{\Gamma}\left(\frac{\partial(\delta y)}{\partial \mathbf{n}} \hat{P}+\frac{\partial \hat{P}}{\partial \mathbf{n}} \delta y\right) d \Gamma .
$$

Furthermore, the divergence theorem implies that

$$
\int_{\Gamma}\left(\frac{\partial(\delta y)}{\partial \mathbf{n}} \hat{P}+\frac{\partial \hat{P}}{\partial \mathbf{n}} \delta y\right) d \Gamma=\int_{\Omega} \operatorname{div} \gamma d \Omega
$$

where $\gamma=(\nabla \delta y) \hat{P}+(\nabla \hat{P}) \delta y$. Therefore $\mathcal{W}(t)=\mathcal{W}_{1}(t)+\mathcal{W}_{2}(t)$, where

$$
\begin{aligned}
& \mathcal{W}_{1}(t)=\int_{\Omega}((I-A(t)) \nabla \delta y \nabla \hat{P}) d \Omega, \\
& \mathcal{W}_{2}(t)=\int_{\Omega} I_{t}\left(B_{t}\right)_{k} \nabla\left(\theta_{k}^{r}-\theta_{k}\right)-e_{k} \nabla \gamma_{k} d \Omega .
\end{aligned}
$$

Observe that $\mathcal{W}_{2}(t)$ can be expressed as

$$
\begin{aligned}
\mathcal{W}_{2}(t) & =\int_{\Omega}\left(I_{t}\left(B_{t}\right)_{k}-e_{k}\right) \nabla\left(\theta_{k}^{r}-\theta_{k}\right)+e_{k} \nabla\left(\theta_{k}^{r}-\theta_{k}-\gamma_{k}\right) d \Omega \\
& =\int_{\Omega}\left(I_{t}\left(B_{t}\right)_{k}-e_{k}\right) \nabla\left(\theta_{k}^{r}-\theta_{k}\right)+\operatorname{div}\left(\theta^{r}-\theta-\gamma\right) d \Omega \\
& =\int_{\Omega}\left(I_{t}\left(B_{t}\right)_{k}-e_{k}\right) \nabla\left(\theta_{k}^{r}-\theta_{k}\right) d \Omega+\int_{\Gamma}\left(\theta^{r}-\theta-\gamma\right) \cdot \mathbf{n} d \Gamma,
\end{aligned}
$$

where

and

$$
\left(\theta^{r}-\theta-\gamma\right) \cdot \mathbf{n}=\left(B_{t}-I\right) \frac{\partial(\delta y)}{\partial \mathbf{n}} \hat{P}+\left(B_{t}-I\right) \frac{\partial \hat{P}}{\partial \mathbf{n}} \delta y
$$

$$
\theta^{r}-\theta=\left(B_{t} \nabla \delta y\right) \hat{P}+\left(B_{t} \nabla \hat{P}\right) \delta y
$$

Let

$$
\mathcal{W}_{2,1}(t):=\int_{\Omega}\left(I_{t}\left(B_{t}\right)_{k}-e_{k}\right) \nabla\left(\theta_{k}^{r}-\theta_{k}\right) d \Omega
$$

and

$$
\mathcal{W}_{2,2}(t):=\int_{\Gamma}\left(\theta^{r}-\theta-\gamma\right) \cdot \mathbf{n} d \Gamma
$$


Then the following estimates hold,

$$
\begin{aligned}
\left|\mathcal{W}_{2,1}(t)\right| & \leq\left\|\left(I_{t}\left(B_{t}\right)_{k}-e_{k}\right)\right\|_{\infty}\left\|\theta_{k}^{r}-\theta_{k}\right\|_{H^{1}} \\
& \leq\left\|\left(I_{t}\left(B_{t}\right)_{k}-e_{k}\right)\right\|_{\infty}\left(\left\|B_{t}\right\|_{\infty}\|\delta y\|_{H^{2}}\|\hat{P}\|_{H^{1}}+\left\|B_{t}\right\|_{\infty}\|\hat{P}\|_{H^{2}}\|\delta y\|_{H^{2}}\right), \\
\left|\mathcal{W}_{2,2}(t)\right| & \left.\leq 2 \|\left(B_{t}\right)-I\right) \|_{\infty}\left(\|\delta y\|_{H^{2}}\|\hat{P}\|_{H^{2}}\right) \\
\left|\mathcal{W}_{1}(t)\right| & \leq \| I-A(t)) \|_{\infty}\left(\|\delta y\|_{H^{2}}\|\hat{P}\|_{H^{2}}\right) .
\end{aligned}
$$

Analogously, we can find the estimates for $\mathcal{S}(t)$. The result follows from Lemma 3.5, Lemma 4.1, and the differentiability of the mappings $t \mapsto A(t), t \mapsto I_{t}$, and $t \mapsto B_{t}$.

The following lemma will become important in what follows.

Lemma 4.3. Let $p_{\phi}:=\left(p \circ T_{t}^{-1}\right), \hat{\Sigma}_{\phi}:=\left(\hat{\Sigma} \circ T_{t}^{-1}\right), y_{\phi}:=y \circ T_{t}^{-1}, \hat{P}_{\phi}:=\left(\hat{P} \circ T_{t}^{-1}\right)$ and

$$
\begin{array}{r}
\tilde{G}\left(\Omega_{t}(\mathbf{W}), y, p, \hat{P}, \hat{\Sigma}\right):=\int_{\Omega_{t}(\mathbf{W})} f \hat{P}_{\phi}-\nabla y_{\phi} \cdot \nabla \hat{P}_{\phi}+\operatorname{div}\left(\nabla y_{\phi} \hat{P}_{\phi}+\nabla \hat{P}_{\phi} y_{\phi}\right) d \Omega_{t}(\mathbf{W}) \\
+\int_{\Omega_{t}(\mathbf{W})}\left(y_{\phi}-y_{d}\right) \hat{\Sigma}_{\phi}-\nabla p_{\phi} \cdot \nabla \hat{\Sigma}_{\phi}+\operatorname{div}\left(\nabla p_{\phi} \hat{\Sigma}_{\phi}+\nabla \hat{\Sigma}_{\phi} p_{\phi}\right) d \Omega_{t}(\mathbf{W}) .
\end{array}
$$

Then the partial derivative of $\tilde{G}\left(\Omega_{t}(\mathbf{W}), y, p, \hat{P}, \hat{\Sigma}\right)$ with respect to $t$ is given by $\left.\partial_{t} \tilde{G}\right|_{t=0}=-\int_{\Omega} \operatorname{div}([\nabla \dot{y} \cdot \nabla p+\nabla y \cdot \nabla \dot{p}] \mathbf{V}) d \Omega+\int_{\Gamma}(\nabla y \cdot \nabla \hat{P}+\nabla p \cdot \nabla \hat{\Sigma}) \mathbf{W} \cdot \mathbf{n} d \Gamma$, where $\dot{y}=-\nabla y \cdot \mathbf{W} \in H^{1}(\Omega), \dot{p}=-\nabla p \cdot \mathbf{W} \in H^{1}(\Omega), \dot{\hat{P}}=-\nabla \hat{P} \cdot \mathbf{W} \in H^{1}(\Omega)$, and $\dot{\hat{\Sigma}}=-\nabla \hat{\Sigma} \cdot \mathbf{W} \in H^{1}(\Omega)$.

Proof. Since $p, \hat{\Sigma}, y$ and $\hat{P}$ belong to $H^{2}(\Omega), p_{\phi}, \hat{\Sigma}_{\phi}, y_{\phi}$ and $\hat{P}_{\phi}$ also belong to $H^{2}(\Omega)$ by Lemma 3.2(2). Furthermore, the derivatives of $p, \hat{\Sigma}, y$, and $\hat{P}$, with respect to $t$ at $t=0$ exist in $H^{1}(\Omega)$ by Lemma $3.2(3)$ and are given by $\dot{p}, \dot{\hat{\Sigma}}, \dot{y}$, and $\dot{\hat{P}}$, respectively. Thus, using Lemma $3.1(1)$ with $g(x, t)=f \hat{P}_{\phi}-\nabla y_{\phi} \cdot \nabla \hat{P}_{\phi}+\operatorname{div}\left(\nabla y_{\phi} \hat{P}_{\phi}+\nabla \hat{P}_{\phi} y_{\phi}\right)+$ $\left(y_{\phi}-y_{d}\right) \hat{\Sigma}_{\phi}-\nabla p_{\phi} \cdot \nabla \hat{\Sigma}_{\phi}+\operatorname{div}\left(\nabla p_{\phi} \hat{\Sigma}_{\phi}+\nabla \hat{\Sigma}_{\phi} p_{\phi}\right)$, we obtain

$$
\begin{aligned}
\left.\partial_{t} \tilde{G}\right|_{t=0}= & \int_{\Gamma}(f \hat{P}-\nabla y \cdot \nabla \hat{P}+\operatorname{div}(\nabla y \hat{P}+\nabla \hat{P} y)) \mathbf{W} \cdot \mathbf{n} d \Gamma \\
& +\int_{\Gamma}\left(\left(y-y_{d}\right) \hat{\Sigma}-\nabla p \cdot \nabla \hat{\Sigma}+\operatorname{div}(\nabla p \hat{\Sigma}+\nabla \hat{\Sigma} p)\right) \mathbf{W} \cdot \mathbf{n} d \Gamma \\
& +\int_{\Omega}(f \dot{\hat{P}}-\nabla y \cdot \nabla \dot{\hat{P}}+\operatorname{div}(\nabla y \dot{\hat{P}}+\nabla \dot{\hat{P}} y)) d \Omega \\
& +\int_{\Omega}\left(\left(y-y_{d}\right) \dot{\hat{\Sigma}}-\nabla p \cdot \nabla \dot{\hat{\Sigma}}+\operatorname{div}(\nabla p \dot{\hat{\Sigma}}+\nabla \dot{\hat{\Sigma}} p)\right) d \Omega
\end{aligned}
$$




$$
\begin{aligned}
& +\int_{\Omega}(\dot{y} \hat{\Sigma}-\nabla \dot{y} \cdot \nabla \hat{P}+\operatorname{div}(\nabla \dot{y} \hat{P}+\nabla \hat{P} \dot{y})) d \Omega \\
& +\int_{\Omega}(-\nabla \dot{p} \cdot \nabla \hat{\Sigma}+\operatorname{div}(\nabla \dot{p} \hat{\Sigma}+\nabla \hat{\Sigma} \dot{p})) d \Omega .
\end{aligned}
$$

Note that (4.8), (4.9) imply that the third and forth integrals in (4.14) vanish, i.e.,

$$
\begin{gathered}
\langle E(y, \Omega), \dot{\hat{P}}\rangle_{\Upsilon}=\int_{\Omega}(f \dot{\hat{P}}-\nabla y \cdot \nabla \dot{\hat{P}}+\operatorname{div}(\nabla y \dot{\hat{P}}+\nabla \dot{\hat{P}} y)) d \Omega=0, \\
\langle E(p, y, \Omega), \dot{\hat{\Sigma}}\rangle_{\Upsilon}=\int_{\Omega}\left(\left(y-y_{d}\right) \dot{\hat{\Sigma}}-\nabla p \cdot \nabla \dot{\hat{\Sigma}}+\operatorname{div}(\nabla p \dot{\hat{\Sigma}}+\nabla \dot{\hat{\Sigma}} p)\right) d \Omega=0,
\end{gathered}
$$

respectively. In addition, utilizing (4.7) with $\tilde{p}=\dot{p}$ and $\tilde{y}=\dot{y}$, one obtains for the last two integrals in (4.14)

$$
\begin{aligned}
\int_{\Omega}(\dot{y} \hat{\Sigma}-\nabla \dot{y} \cdot \nabla \hat{P}+\operatorname{div}(\nabla \dot{y} \hat{P}+\nabla \hat{P} \dot{y})) d \Omega & =-\int_{\Omega} \operatorname{div}([\nabla \dot{y} \cdot \nabla p] \mathbf{V}) d \Omega, \\
\int_{\Omega}(-\nabla \dot{p} \cdot \nabla \hat{\Sigma}+\operatorname{div}(\nabla \dot{p} \hat{\Sigma}+\nabla \hat{\Sigma} \dot{p})) d \Omega & =-\int_{\Omega} \operatorname{div}([\nabla y \cdot \nabla \dot{p}] \mathbf{V}) d \Omega .
\end{aligned}
$$

Furthermore, observe that

$$
\int_{\Gamma} \operatorname{div}(\nabla y \hat{P}+\nabla \hat{P} y) d \Gamma=\int_{\Gamma} 2 \nabla y \cdot \nabla \hat{P}+\Delta y \hat{P}+\Delta \hat{P} y d \Gamma .
$$

Since $\Delta y+f=0$ on $\Gamma$, and $y=0$ on $\Gamma$, we have for the first integral in (4.14)

$$
\int_{\Gamma}(f \hat{P}-\nabla y \cdot \nabla \hat{P}+\operatorname{div}(\nabla y \hat{P}+\nabla \hat{P} y)) \mathbf{W} \cdot \mathbf{n} d \Gamma=\int_{\Gamma} \nabla y \cdot \nabla \hat{P} \mathbf{W} \cdot \mathbf{n} d \Gamma .
$$

Analogously for the second integral,

$$
\int_{\Gamma}\left(\left(y-y_{d}\right) \hat{\Sigma}-\nabla p \cdot \nabla \hat{\Sigma}+\operatorname{div}(\nabla p \hat{\Sigma}+\nabla \hat{\Sigma} p)\right) \mathbf{W} \cdot \mathbf{n} d \Gamma=\int_{\Gamma} \nabla p \cdot \nabla \hat{\Sigma} \mathbf{W} \cdot \mathbf{n} d \Gamma .
$$

Consequently,

$\left.\partial_{t} \tilde{G}\right|_{t=0}=-\int_{\Omega} \operatorname{div}([\nabla \dot{y} \cdot \nabla p+\nabla y \cdot \nabla \dot{p}] \mathbf{V}) d \Omega+\int_{\Gamma}(\nabla y \cdot \nabla \hat{P}+\nabla p \cdot \nabla \hat{\Sigma}) \mathbf{W} \cdot \mathbf{n} d \Gamma$.

4.2. Shape Hessian. In this subsection we establish the expression for the shape Hessian for the cost functional $J$. In what follows, we shall make use of the the summation convention. For instance, where necessary, the divergence of a vectorial function $\psi$ shall be expressed as

$$
(\operatorname{div} \boldsymbol{\psi})=e_{i} \nabla \psi_{i}
$$

where $e_{i}$ stands for the i-th canonical basis vector in $\mathbb{R}^{d}$. Furthermore, we shall make use of the transformation of the divergence from $\Omega_{t}(\mathbf{W})$ to $\Omega$. Using (3.6), 
and the summation convection in (4.15), one can express this transformation as

$$
\left(\operatorname{div} \boldsymbol{\psi}_{t}\right) \circ T_{t}=D \psi_{i}^{t} B_{t}^{T} e_{i}=\left(B_{t}\right)_{i} \nabla \psi_{i}^{t},
$$

where $\left(B_{t}\right)_{i}$ denotes the i-th row of $B_{t}=\left(D T_{t}\right)^{-T}$.

Theorem 4.1. The shape Hessian of $J(\Omega)$ exists and it is given by the expression

$$
d^{2} J(\Omega)(\mathbf{V}, \mathbf{W})=\int_{\Gamma}\left(\nabla y \cdot \nabla \hat{P}+\nabla p \cdot \nabla \hat{\Sigma}+\operatorname{div}\left(\left[\frac{1}{2} y_{d}^{2}+\nabla y \cdot \nabla p\right] \mathbf{V}\right)\right) \mathbf{W} \cdot \mathbf{n} d \Gamma .
$$

Proof. Let $\Delta:=d J\left(\Omega_{t}(\mathbf{W})\right) \mathbf{V}-d J(\Omega) \mathbf{V}, \Theta:=\left[\frac{1}{2} y_{d}^{2}+(\nabla y, \nabla p)\right] \mathbf{V}$, and $\Theta^{t}:=\left[\frac{1}{2} y_{d}^{2}+\left(B_{t} \nabla y^{t}, B_{t} \nabla p^{t}\right)\right] \mathbf{V}$. Then using Lemma 3.3, (3.6) and (4.16), we can express $\Delta$ as

$$
\Delta=\int_{\Omega} I_{t}\left(B_{t}\right)_{k} \nabla \Theta_{k}^{t}-e_{k} \nabla \Theta_{k} d \Omega
$$

We re-write the right hand side of (4.17) such that $\Delta=S(t)+R(t)$, where

$$
\begin{aligned}
S(t) & :=\int_{\Omega} I_{t}\left(\left(B_{t}\right)_{k} \nabla \Theta_{k}^{t}-e_{k} \nabla \Theta_{k}^{r}\right) d \Omega, \\
R(t) & :=\int_{\Omega}\left(I_{t} e_{k} \nabla \Theta_{k}^{r}-e_{k} \nabla \Theta_{k}\right) d \Omega,
\end{aligned}
$$

and

$$
\Theta^{r}:=\left[\frac{1}{2} y_{d}^{2}+\left(\nabla y^{t}, \nabla p^{t}\right)\right] \mathbf{V}
$$

The task now is to evaluate $\lim _{t \rightarrow 0^{+}}|R(t)| / t+|S(t)| / t$. We will do this in several steps. To this end, the terms on the right hand side of (4.19) and (4.18) are rearranged to obtain $R(t):=R_{1}(t)+R_{2}(t)+R_{3}(t)$ and $S(t):=S_{1}(t)+S_{2}(t)$, respectively, where

$$
\begin{aligned}
R_{1}(t) & =\int_{\Omega}\left(I_{t}-1\right) e_{k} \nabla\left(\Theta_{k}^{r}-\Theta_{k}\right) d \Omega, \quad R_{2}(t)=\int_{\Omega} e_{k} \nabla\left(\Theta_{k}^{r}-\Theta_{k}\right) d \Omega \\
R_{3}(t) & =\int_{\Omega}\left(I_{t}-1\right) e_{k} \nabla \Theta_{k} d \Omega \\
S_{1}(t) & =\int_{\Omega} I_{t}\left(\left(B_{t}\right)_{k} \nabla\left(\Theta_{k}^{t}-\Theta_{k}^{s}\right)-e_{k} \nabla\left(\Theta_{k}^{r}-\Theta_{k}\right)\right) d \Omega \\
S_{2}(t) & =\int_{\Omega} I_{t}\left(\left(B_{t}\right)_{k} \nabla \Theta_{k}^{s}-e_{k} \nabla \Theta_{k}\right) d \Omega
\end{aligned}
$$

and

$$
\Theta^{s}=\left[\frac{1}{2} y_{d}^{2}+\left(B_{t} \nabla y, B_{t} \nabla p\right)\right] \mathbf{V} .
$$

We now evaluate $\lim _{t \rightarrow 0^{+}}\left|R_{1}(t)\right|$. Note that by using the relation $a b-c d=(a-c)(b-$ $d)+(a-c) d+c(b-d)$, with $a=\nabla y^{t}, b=\nabla p^{t}, c=\nabla y$, and $d=\nabla p$, we can express $\Theta^{r}-\Theta$ as

$$
\begin{aligned}
\Theta^{r}-\Theta & =\left[\left(\nabla y^{t}, \nabla p^{t}\right)-(\nabla y, \nabla p)\right] \mathbf{V} \\
& =[(\nabla \delta y, \nabla \delta p)] \mathbf{V}+[(\nabla \delta y, \nabla p)+(\nabla y, \nabla \delta p)] \mathbf{V}
\end{aligned}
$$


where $\delta y=y^{t}-y$ and $\delta p=p^{t}-p$.

Using (4.21), the divergence theorem, the trace theorem and the fact that $\delta y \in$ $H_{0}^{1}(\Omega) \cap H^{2}(\Omega), \delta p \in H_{0}^{1}(\Omega) \cap H^{2}(\Omega)$, we can estimate $\frac{1}{t}\left|R_{1}(t)\right|$ as

$$
\begin{aligned}
\frac{1}{t}\left|R_{1}(t)\right| & \leq \frac{1}{t}\left\|I_{t}-1\right\|_{L^{\infty}} \int_{\Gamma}\left|\frac{\partial(\delta y)}{\partial \mathbf{n}} \frac{\partial(\delta p)}{\partial \mathbf{n}} \mathbf{V} \cdot \mathbf{n}\right| d \Gamma \\
& +\frac{1}{t}\left\|I_{t}-1\right\|_{L^{\infty}} \int_{\Gamma}\left|\left(\frac{\partial(\delta y)}{\partial \mathbf{n}} \frac{\partial p}{\partial \mathbf{n}}+\frac{\partial y}{\partial \mathbf{n}} \frac{\partial(\delta p)}{\partial \mathbf{n}}\right) \mathbf{V} \cdot \mathbf{n}\right| d \Gamma \\
& \leq \frac{1}{t}\left\|I_{t}-1\right\|_{L^{\infty}}\|\delta y\|_{H^{2}(\Omega)}\|\delta p\|_{H^{2}(\Omega)}\|\mathbf{V} \cdot \mathbf{n}\|_{L^{\infty}(\Gamma)} \\
& +\frac{1}{t}\left\|I_{t}-1\right\|_{L^{\infty}}\|\delta y\|_{H^{2}(\Omega)}\|p\|_{H^{2}(\Omega)}\|\mathbf{V} \cdot \mathbf{n}\|_{L^{\infty}(\Gamma)} \\
& +\frac{1}{t}\left\|I_{t}-1\right\|_{L^{\infty}}\|y\|_{H^{2}(\Omega)}\|\delta p\|_{H^{2}(\Omega)}\|\mathbf{V} \cdot \mathbf{n}\|_{L^{\infty}(\Gamma)} .
\end{aligned}
$$

Using Lemma 3.5, Lemma 4.1, and the differentiability of the mapping $t \mapsto I_{t}$, it follows that $\lim _{t \rightarrow 0^{+}} \frac{1}{t}\left|R_{1}(t)\right|=0$.

Next, we evaluate $\lim _{t \rightarrow 0^{+}} \frac{R_{2}(t)}{t}$. Observe that by using (4.15) and (4.21), one can express $R_{2}(t)$ in $(4.20)$ as $R_{2}(t)=\mathcal{X}_{1}(t)+\mathcal{X}_{2}(t)$, where

$$
\begin{aligned}
\mathcal{X}_{1}(t) & :=\int_{\Omega} \operatorname{div}([(\nabla \delta y, \nabla \delta p)] \mathbf{V}) d \Omega, \\
\mathcal{X}_{2}(t) & :=\int_{\Omega} \operatorname{div}([(\nabla \delta y, \nabla p)+(\nabla y, \nabla \delta p)] \mathbf{V}) d \Omega .
\end{aligned}
$$

Using the divergence theorem and the fact that $\delta y, \delta p \in H_{0}^{1}(\Omega) \cap H^{2}(\Omega)$, we can express $\mathcal{X}_{1}(t)$ as

$$
\mathcal{X}_{1}(t)=\int_{\Gamma} \frac{\partial(\delta y)}{\partial \mathbf{n}} \frac{\partial(\delta p)}{\partial \mathbf{n}} \mathbf{V} \cdot \mathbf{n} d \Gamma
$$

and the following estimate holds

$$
\begin{aligned}
\left|\mathcal{X}_{1}(t)\right| & \leq \int_{\Gamma}\left|\frac{\partial(\delta y)}{\partial \mathbf{n}} \frac{\partial(\delta p)}{\partial \mathbf{n}} \mathbf{V} \cdot \mathbf{n}\right| d \Gamma \\
& \leq\left.\left\|\frac{\partial(\delta y)}{\partial \mathbf{n}}\right\|\right|_{L^{2}(\Gamma)}\left\|\frac{\partial(\delta p)}{\partial \mathbf{n}}\right\|_{L^{2}(\Gamma)}\|\mathbf{V} \cdot \mathbf{n}\|_{L^{\infty}(\Gamma)} \\
& \leq\|\delta y\|_{H^{2}(\Omega)}\|\delta p\|_{H^{2}(\Omega)}\|\mathbf{V} \cdot \mathbf{n}\|_{L^{\infty}(\Gamma)}, \quad \text { by the trace theorem }
\end{aligned}
$$

Therefore, by Lemma 3.5 and Lemma 4.1 we have

$$
\lim _{t \rightarrow 0^{+}} \frac{1}{t}\left|\mathcal{X}_{1}(t)\right| \leq \lim _{t \rightarrow 0+} \frac{1}{\sqrt{t}}\|\delta y\|_{H^{2}(\Omega)} \frac{1}{\sqrt{t}}\|\delta p\|_{H^{2}(\Omega)}\|\mathbf{V} \cdot \mathbf{n}\|_{L^{\infty}(\Gamma)}=0 .
$$


Next, we need to evaluate $\lim _{t \rightarrow 0^{+}} \frac{\mathcal{X}_{2}(t)}{t}$. Using $(4.7)$, we can express $\mathcal{X}_{2}(t)$ as

$$
\begin{aligned}
\mathcal{X}_{2}(t)=\int_{\Omega} & (\nabla \delta y \cdot \nabla \hat{P}-\delta y \hat{\Sigma}) d \Omega-\int_{\Gamma}\left(\frac{\partial \delta y}{\partial \mathbf{n}} \hat{P}+\frac{\partial \hat{P}}{\partial \mathbf{n}} \delta y\right) d \Gamma \\
& +\int_{\Omega}(\nabla \delta p \cdot \nabla \hat{\Sigma}) d \Omega-\int_{\Gamma}\left(\frac{\partial(\delta p)}{\partial \mathbf{n}} \hat{\Sigma}+\frac{\partial \hat{\Sigma}}{\partial \mathbf{n}}(\delta p)\right) d \Gamma
\end{aligned}
$$

where $(y, p) \in H^{2}(\Omega) \times H^{2}(\Omega)$ satisfy (1.2) and (3.10). Observe that $\mathcal{X}_{2}(t)$ can be expressed as

$$
\begin{aligned}
\mathcal{X}_{2}(t)= & \left\langle\tilde{E}\left(y^{t}, t\right)-\tilde{E}(y, t)-\left(E\left(y^{t}, \Omega\right)-E(y, \Omega)\right), \hat{P}\right\rangle_{\Upsilon} \\
& +\left\langle\tilde{E}\left(p^{t}, y^{t}, t\right)-\tilde{E}(p, y, t)-\left(E\left(p^{t}, y^{t}, \Omega\right)-E(p, y, \Omega)\right), \hat{\Sigma}\right\rangle_{\Upsilon} \\
& +\langle\tilde{E}(p, y, t), \hat{\Sigma}\rangle_{\Upsilon}-\langle\tilde{E}(p, y, 0), \hat{\Sigma}\rangle_{\Upsilon}+\langle\tilde{E}(y, t), \hat{P}\rangle_{\Upsilon}-\langle\tilde{E}(y, 0), \hat{P}\rangle_{\Upsilon}
\end{aligned}
$$

where the extra terms $\left\langle\tilde{E}\left(y^{t}, t\right), \hat{P}\right\rangle_{\Upsilon},\langle\tilde{E}(y, 0), \hat{P}\rangle_{\Upsilon},\left\langle\tilde{E}\left(p^{t}, y^{t}, t\right), \hat{\Sigma}\right\rangle_{\Upsilon}$, and $\langle\tilde{E}(p, y, 0), \hat{\Sigma}\rangle_{\Upsilon}$ introduced, vanish by $(4.10),(4.11)$ and the fact that $\langle\tilde{E}(y, 0), \hat{P}\rangle_{\Upsilon}=\langle\tilde{E}(y, \Omega), \hat{P}\rangle_{\Upsilon}$, and $\langle\tilde{E}(p, y, 0), \hat{\Sigma}\rangle_{\Upsilon}=\langle E(p, y, \Omega), \hat{\Sigma}\rangle_{\Upsilon}$. Utilizing the notation used in Lemma 4.2 , we obtain

$$
\begin{aligned}
\mathcal{X}_{2}(t)= & \mathcal{W}(t)+\mathcal{S}(t)+\langle\tilde{E}(p, y, t), \hat{\Sigma}\rangle_{\Upsilon}-\langle\tilde{E}(p, y, 0), \hat{\Sigma}\rangle_{\Upsilon} \\
& +\langle\tilde{E}(y, t), \hat{P}\rangle_{\Upsilon}-\langle\tilde{E}(y, 0), \hat{P}\rangle_{\Upsilon}
\end{aligned}
$$

Using Lemma 4.2, we obtain

$$
\lim _{t \rightarrow 0+} \frac{1}{t} \mathcal{X}_{2}(t)=\left.\frac{d}{d t} \tilde{E}(y, \hat{P} ; t)\right|_{t=0}+\left.\frac{d}{d t} \tilde{E}(p, y, \hat{\Sigma} ; t)\right|_{t=0} .
$$

The expression on the right hand side of (4.22) can be computed by transforming $y, \hat{P}, p, \hat{\Sigma}$ defined in $\Omega$ back to $\Omega_{t}(\mathbf{W})$ via $y \mapsto y \circ T_{t}^{-1}, p \mapsto p \circ T_{t}^{-1}, \hat{P} \mapsto \hat{P} \circ T_{t}^{-1}$ and $\hat{\Sigma} \mapsto \hat{\Sigma} \circ T_{t}^{-1}$. Utilizing Lemma 4.3, one obtains

$$
\begin{aligned}
\lim _{t \rightarrow 0+} \frac{1}{t} \mathcal{X}_{2}(t)= & \left.\partial_{t} \tilde{G}\right|_{t=0} \\
= & -\int_{\Omega} \operatorname{div}([\nabla \dot{y} \cdot \nabla p+\nabla y \cdot \nabla \dot{p}] \mathbf{V}) d \Omega \\
& +\int_{\Gamma}(\nabla y \cdot \nabla \hat{P}+\nabla p \cdot \nabla \hat{\Sigma}) \mathbf{W} \cdot \mathbf{n} d \Gamma .
\end{aligned}
$$

We shall revisit $R_{3}(t)$ later on. Let us now provide an estimate for $\left|S_{1}(t)\right| / t$. Observe that

$$
\begin{aligned}
S_{1}(t) & =\int_{\Omega} I_{t}\left(\left(\left(B_{t}\right)_{k}-e_{k}\right) \nabla\left(\Theta_{k}^{t}-\Theta_{k}^{s}\right)+e_{k} \nabla \mathcal{Q}_{k}\right) d \Omega \\
& =\int_{\Omega} I_{t}\left(\operatorname{div}\left(\left(B_{t}-I\right)\left(\Theta^{t}-\Theta^{s}\right)\right)+\operatorname{div} \mathcal{Q}\right) d \Omega
\end{aligned}
$$


where

$$
\begin{aligned}
\mathcal{Q}:=\Theta^{t}-\Theta^{s}-\Theta^{r}+\Theta= & {\left[\left(B_{t}-I\right) \nabla \delta y, B_{t} \nabla p^{t}\right)+\left(\nabla \delta y,\left(B_{t}-I\right) \nabla p^{t}\right) } \\
& +\left(B_{t} \nabla y,\left(B_{t}-I\right) \nabla \delta p\right) \\
& \left.+\left(\left(B_{t}-I\right) \nabla y,\left(B_{t}-I\right) \nabla \delta p\right)\right] \mathbf{V},
\end{aligned}
$$

and

$$
\Theta^{t}-\Theta^{s}=\left[\left(B_{t} \nabla \delta y, B_{t} \nabla p^{t}\right)+\left(B_{t} \nabla y, B_{t} \nabla \delta p\right)\right] \mathbf{V}
$$

Hence we have

$$
\left|\frac{1}{t} S_{1}(t)\right| \leq\left.|| I_{t}\right|_{L^{\infty}(\Omega)} \int_{\Omega}\left|\operatorname{div}\left(\frac{1}{t}\left(B_{t}-I\right)\left(\Theta^{t}-\Theta^{s}\right)+\mathcal{Q}\right)\right| d \Omega .
$$

Using the divergence theorem, and the trace theorem, we obtain the following estimate

$$
\left|\frac{1}{t} S_{1}(t)\right| \leq\left\|I_{t}\right\|_{L^{\infty}(\Omega)}|| \mathbf{V} \cdot \mathbf{n} \|_{L^{\infty}(\Gamma)}(\mathcal{P}(t)+\mathcal{R}(t))
$$

where

$$
\mathcal{P}(t):=\left\|\frac{1}{t}\left(B_{t}-I\right)\right\|_{L^{\infty}}\left\|B_{t}\right\|_{L^{\infty}}^{2}\left(\|\delta y\|_{H^{2}(\Omega)}\left\|p^{t}\right\|_{H^{2}(\Omega)}+\|y\|_{H^{2}(\Omega)}\|\delta p\|_{H^{2}(\Omega)}\right),
$$

and

$$
\begin{aligned}
\mathcal{R}(t):= & \left\|\frac{1}{t}\left(B_{t}-I\right)\right\|_{L^{\infty}}\left\|B_{t}\right\|_{L^{\infty}}\left(\|\delta y\|_{H^{2}(\Omega)}\left\|p^{t}\right\|_{H^{2}(\Omega)}+\|y\|_{H^{2}(\Omega)}\|\delta p\|_{H^{2}(\Omega)}\right) \\
& +\left\|\left(B_{t}-I\right)\right\|_{L^{\infty}}\|\delta y\|_{H^{2}(\Omega)}\left\|p^{t}\right\|_{H^{2}(\Omega)} .
\end{aligned}
$$

Using Lemma 3.5, Lemma 4.1, and the differentiability of the mapping $t \mapsto B_{t}$, one obtains $\lim _{t \rightarrow 0^{+}}\left|\frac{1}{t} S_{1}(t)\right|=0$.

We combine the remaining expressions, i.e., $R_{3}(t)$ and $S_{2}(t)$ into $Q(t)$ such that $Q(t):=S_{2}(t)+R_{3}(t)$. Then $Q(t)$ can be expressed as

$$
Q(t)=\int_{\Omega} I_{t}\left(B_{t}\right)_{k} \nabla \Theta_{k}^{s}-e_{k} \nabla \Theta_{k} d \Omega
$$

Next, we evaluate $\lim _{t \rightarrow 0^{+}} \frac{Q(t)}{t}$. Using Lemma 3.3, (4.15), (4.16), and (3.5), we obtain

$$
\begin{aligned}
Q(t) & =\int_{\Omega_{t}(\mathbf{W})}\left(B_{t}\right)_{k} \nabla \Theta_{k}^{s} \circ T_{t}^{-1} d \Omega_{t}(\mathbf{W})-\int_{\Omega} e_{k} \nabla \Theta_{k} d \Omega \\
& =\int_{\Omega_{t}(\mathbf{W})} \operatorname{div}\left(\Theta^{s} \circ T_{t}^{-1}\right) d \Omega_{t}(\mathbf{W})-\int_{\Omega} \operatorname{div} \Theta d \Omega .
\end{aligned}
$$


Dividing the expression in (4.24) by $t$ and making use of (3.6), the definition of the shape derivative, Lemma 3.1(1), and Lemma 3.2(3), we obtain

$$
\begin{aligned}
\lim _{t \rightarrow 0^{+}} \frac{Q(t)}{t} & =\left.\frac{d}{d t} \int_{\Omega_{t}(\mathbf{W})} \operatorname{div}\left(\left[\frac{1}{2} y_{d}^{2}+\nabla y_{\phi} \cdot \nabla p_{\phi}\right] \mathbf{V}\right) d \Omega_{t}(\mathbf{W})\right|_{t=0} \\
& =\int_{\Omega} \operatorname{div}([\nabla \dot{y} \cdot \nabla p+\nabla y \cdot \nabla \dot{p}] \mathbf{V}) d \Omega \\
& +\int_{\Gamma} \operatorname{div}\left(\left[\frac{1}{2} y_{d}^{2}+\nabla y \cdot \nabla p\right] \mathbf{V}\right) \mathbf{W} \cdot \mathbf{n} d \Gamma
\end{aligned}
$$

Collecting the results in (4.23) and (4.25), we obtain

$$
\begin{aligned}
d^{2} J(\Omega)(\mathbf{V}, \mathbf{W}) & =\lim _{t \rightarrow 0^{+}} \frac{Q(t)}{t}+\lim _{t \rightarrow 0^{+}} \frac{\mathcal{X}_{2}(t)}{t} \\
& =\int_{\Gamma}\left(\nabla y \cdot \nabla \hat{P}+\nabla p \cdot \nabla \hat{\Sigma}+\operatorname{div}\left(\left[\frac{1}{2} y_{d}^{2}+\nabla y \cdot \nabla p\right] \mathbf{V}\right)\right) \mathbf{W} \cdot \mathbf{n} d \Gamma
\end{aligned}
$$

Remark 4.1. The proof of Theorem 4.1 reveals that the essential ingredients for establishing the expression for the shape Hessian of cost functional $J$ are the properties of Lemma 3.5 and Lemma 4.1.

Remark 4.2. The method and the associated computation we presented can be applied to a large class of boundary value problems and a general cost functional $J(u, \Omega, \Gamma)$ depending, besides $u$ and $\Omega$, on a codimension one manifold $\Gamma$. With respect to the latter, suppose that the shape derivative of $J(u, \Omega, \Gamma)$ exists and can be expressed in the form

$$
d J(\Omega)(\mathbf{V})=\int_{\Gamma} g(\Omega) \mathbf{V} \cdot \mathbf{n} d \Gamma
$$

where $g(\Omega) \in H^{3 / 2}(\Gamma)$. Then there exists an extension of $g(\Omega)$ denoted by $Q(\Omega) \in$ $H^{2}(\Omega)$ such that

$$
d J(\Omega)(\mathbf{V})=\int_{\Omega} \operatorname{div}(Q(\Omega) \mathbf{V}) d \Omega .
$$

Here $Q(\Omega)$ depends on the state and adjoint variables $u$ and $p$, respectively. If $u$ and $p$ are Hölder continuous with exponent greater or equal $1 / 2$ with respect to geometrical perturbations, then one can derive the shape Hessian of $J$ using our approach.

\section{REFERENCES}

[Bur04] M. Burger. Levenberg-Marquardt level set methods for inverse obstacle problems. Inverse Problems, 20:259-282, 2004.

[DZ91a] M. Delfour and J-P. Zolesio. Anatomy of the shape Hessian. Ann. Mat. Pur.appl., 159:315-339, 1991. 
[DZ91b] M. Delfour and J-P. Zolesio. Velocity method and Lagrangian formulation for the computation of the shape Hessian. SIAM Journal on Control and Optimization, 29(6):14141442,1991

[DZ01] M. C. Delfour and J. P. Zolésio. Shapes and geometries: analysis, differential calculus, and optimization. Society for Industrial and Applied Mathematics, Philadelphia, PA, USA, 2001.

[Eva98] L. C. Evans. Partial Differential Equations. American Mathematical Society, 1998.

[Fuj87] N. Fujii. Second variation and its application in domain optimization problem. in control of distributed parameter systems, Proceedings of the 4th IFAC symposium, Pergamon Press, 1987.

[GR86] V. Girault and P. A. Raviart. Finite Element Methods for Navier-Stokes. SpringerVerlag, Berlin, 1986.

$\left[\mathrm{HIK}^{+} 09\right]$ J. Haslinger, K. Ito, T. Kozubek, K. Kunisch, and G. Peichl. On the shape derivative for problems of Bernoulli type. Interfaces and Free Boundaries, 11(2):317-330, 2009.

[HP91] A. Henrot and M. Pierre. About critical points of the energy in electromagnetic shaping problem. In: Boundary Control and Boundary Variation, J-P. Zolesio (ed.) Lecture notes in Control and Information Sciences, 178:238-252, 1991.

[IKP06] K. Ito, K. Kunisch, and G. Peichl. Variational approach to shape derivatives for a class of Bernoulli problems. Journal of Mathematical Analysis and Applications, 314(1):126149, 2006.

[IKP08] K. Ito, K. Kunisch, and G. Peichl. Variational approach to shape derivatives. ESAIM: Control, Optimisation and Calculus of Variations, 14:517-539, 2008.

[Sim89] J. Simon. Second variations for domain optimization problems. In: Control and Estimation of Distributed Parameter Systems, F. Kappel et al., eds, International Ser. Numer. Math., 91:361-378, 1989.

[SZ92] J. Sokolowski and J. P. Zolésio. Introduction to Shape Optimization. Shape Sensitivity Analysis. Springer-Verlag, 1992.

[Ti197] T. Tilhonen. Shape optimization and trial methods for free boundary problems. Mathematical Modelling and Numerical Analysis, 31:805-825, 1997.

(H. Kasumba) Johann Radon Institute for Computational and Applied Mathematics, Austrian Academy of Sciences, Altenbergerstrasse 69, A-4040 Linz, Austria

E-mail address: henry.kasumba@oeaw.at,

(K. Kunisch) Institut für Mathematik und Wissenschaftliches Rechnen, KarlFranzens Universität Graz, Heinrichstrasse 36, A-8010 Graz, Austria

E-mail address: karl.kunisch@uni-graz.at 